\title{
Phänomenologie und Ethnomethodologie
}

Nach einer einprägsamen Formel von Thomas Luckmann (1979: 196ff) ist Phänomenologie Philosophie, Soziologie dagegen Wissenschaft. Die Perspektive der Phänomenologie sei egologisch, jene der Wissenschaft kosmologisch. Die Methode der Phänomenologie sei reflexiv, jene der Wissenschaft induktiv. Das Ziel der Phänomenologie sei es, »die universalen Strukturen subjektiver Orientierung in der Welt zu beschreiben«, das Hauptziel der Wissenschaft dagegen, "die allgemeinen Merkmale der objektiven Welt zu erklären«(ibid.). Luckmann interpretiert die phänomenologische Lebensweltanalyse von Alfred Schütz daher als "Protosoziologie«, als "mathesis universalis", von der er sich die Lösung eines Fundamentalproblems der Sozialwissenschaften verspricht: des Problems der Vergleichbarkeit historischer Daten (Luckmann 1980: 52). Wie das Verhältnis von Phänomenologie und Soziologie aussehen kann, hat er zusammen mit Peter L. Berger in Die gesellschaftliche Konstruktion der Wirklichkeit demonstriert: Zentrale Einsichten von Schütz wurden als "präsoziologische« Grundlage verwendet, um ihre Neukonzeption der Wissenssoziologie phänomenologisch zu unterfüttern (Berger \& Luckmann 1970).

Eine völlig andere Auffassung des Verhältnisses von Phänomenologie und Soziologie hat Harold Garfinkel mit seiner Ethnomethodologie entwickelt. Er interpretierte Schütz’ Lebensweltanalyse nicht als protosoziologische Grundlagentheorie, sondern vielmehr als alternatives soziologisches Paradigma zur Erklärung des Problems sozialer Ordnung. In der Ethnomethodologie wurden Phänomenologie und Soziologie also miteinander verschmolzen. Das Verhältnis von Phänomenologie und Ethnomethodologie ist schon verschiedentlich reflektiert worden (vgl. v. a. Eberle 1984, Heritage 1984, Sharrock \& Anderson 1989). Die Behauptung ist nicht übertrieben, dass die Ethnomethodologie ohne Phänomenologie gar nie entstanden wäre. Es gibt nun mindestens zwei Gründe, erneut einen Blick auf das Verhältnis zu werfen. Erstens kann die Genese der Ethnomethodologie dank neuer Publikationen präziser rekonstruiert werden, als dies in den 1980er Jahren möglich war. Zweitens fällt auf, dass die frühen Ethnomethodologen sich alle mit Schütz auseinandergesetzt und immer wieder auf sein Werk Bezug genommen haben. Bei den heutigen Ethnomethodologen ist dies nicht mehr das Fall. Selbst Garfinkel (2002) bezieht sich in seinen neueren Schriften kaum mehr auf Schütz, und Michael Lynch (1993) konstatiert, die (falschen) Schützschen Grundannahmen seien inzwischen überwunden. Hat die heutige Ethnomethodologie überhaupt noch eine Beziehung zur Phänomenologie?

\section{Garfinkels Auseinandersetzung mit der Phänomenologie}

In seinem Hauptwerk Studies in Ethnomethodology nennt Garfinkel (1967: ix) einen Soziologen und drei Phänomenologen als seine intellektuellen Mentoren: Talcott Parsons, Alfred Schütz, Aron Gurwitsch und Edmund Husserl. Mit Phänomenologie kam er bereits während seines Master-Studiums in Kontakt. Zunächst hatte er - mit der Perspektive, in seines Vaters Möbelgeschäft einzusteigen - an der Universität seiner Heimatstadt Newark in New Jersey Betriebswirtschaftslehre studiert und mit dem Bachelor-Degree abgeschlossen. Von 1939 bis 1942 absolvierte er an der University of North Carolina in Chapel Hill ein Master-Studium in Soziologie, während dessen er mit Kollegen von der Philosophischen Abteilung Bekanntschaft schloss und Texte von Husserl, Gurwitsch und Gestalt-Psychologen las (Garfinkel 2002: 82ff, Rawls 2002: 11ff). Laut einem Interview mit Psathas (im Druck) nahm Garfinkel auch phänomenologische Texte in die Armee mit, in der er von 1942 bis 1946 diente. 
1946 begann er sein Ph.D.-Studium bei Talcott Parsons an der Harvard University, wo er bis 1951 blieb. Während dieser Zeit pflegte er Beziehungen zu Aron Gurwitsch, den er regelmäßig in dessen Haus in Cambridge, Mass., besuchte, sowie zu Alfred Schütz, den er häufig in New York für Tutoriatsabende traf (Garfinkel 2002: 84). Im Rahmen dieser Kontakte wurde sein Interesse an phänomenologischen Fragen immer größer und gründlicher (Rawls 2002: 15), und es schlug sich auch deutlich in den zwei Arbeiten dieser Zeit nieder, die beide im Spannungsfeld zwischen der Phänomenologie einerseits und dem Parsons'schen Strukturfunktionalismus andererseits entstanden: in der Studie Seeing Sociologically (Garfinkel [1948] 2006) und in seiner Dissertation The Perception of the Other: A Study in Social Order (Garfinkel 1952). Während die Dissertationsschrift unveröffentlicht blieb, bei der Harvard University Library aber als Kopie auf Microfiche bestellt werden kann, wurde eine 1948 entstandene, 117 Seiten lange Vorstudie zu einer Dissertation mit dem umständlichen Titel Prospectus for an Explanatory Study of Communicative Effort and the Modes of Understanding in Selected Types of Dyadic Relationship erst kürzlich von Anne Rawls entdeckt und veröffentlicht (Garfinkel 2006, Rawls 2006). Dieser Text, der seinerzeit u. a. auch von Erving Goffman, Anselm Strauss und Harvey Sacks gelesen wurde (Rawls 2006: 2), skizziert bereits die Grundlinien des ethnomethodologischen Forschungsprogramms. Dessen Einlösung, so bemerkt Lemert (2006) im Vorwort, konnte eine Dissertation nicht leisten, sondern nahm ein ganzes Forscherleben in Anspruch. Rawls (2006: 2) qualifiziert diese Schrift als "a dissertation that was never written", sie kann m. E. aber auch als Vorstudie zu seiner Dissertation gewertet werden, welche viele inhaltliche Übereinstimmungen aufweist. Beide Schriften enthalten ausführliche Erörterungen über die Adäquanz theoretischer Prämissen und soziologischer Konzepte, also das, was sich viele von Garfinkel an Klärung immer gewünscht hatten und was dieser später hartnäckig verweigerte. In beiden Schriften wird der enorme Einfluss deutlich, den die Phänomenologie bei der Entstehung der Ethnomethodologie hatte.

\section{Von der egologischen Perspektive zur Beobachterperspektive}

Husserls Phänomenologie nimmt das ego-cogito-cogitatum zum Ausgangspunkt, also die Intentionalität des subjektiven Bewusstseins, und analysiert die Gegebenheitsweisen der Phänomene in ihren noetischen und noematischen Aspekten. Der Sinn der Phänomene konstituiert sich durch polythetische Bewusstseinsleistungen in der Zeitlichkeit des Bewusstseinsstroms. Schütz hat diese Analysen der Sinnkonstitution aufgenommen und fortgeführt, um die handlungstheoretische Grundlegung der Verstehenden Soziologie durch Max Weber philosophisch zu begründen. Dabei ging es ihm darum, die Sinnkategorie sowie die Strukturen der Lebenswelt zu klären. In Bezug auf Weber wies er auf die Notwendigkeit hin, die Zeitlichkeit sowie die Perspektivität der Sinnkonstitution zu beachten: Insbesondere soll zwischen verschiedenen Sinnschichten, zwischen subjektiver und objektiven Sinnzusammenhängen, zwischen Selbstdeutung und Fremddeutung sowie zwischen verschiedenen zeitlichen und räumlichen Gegebenheitsweisen unterschieden werden. Im Übrigen war Schütz (2004: 75) überzeugt, dass es Weber gelungen war, den Ansatzpunkt jeder echten Theorie der Sozialwissenschaften endgültig zu bestimmen.

In Seeing Sociologically greift Garfinkel (2006 [1948]) diese Analysen auf. Im Unterschied zu Schütz war er nicht an der Weberschen Soziologie, sondern am Parsons'schen Theorierahmen orientiert, und er setzte sich zum Ziel, unter Rückgriff auf die Phänomenologie eine neue soziologische Perspektive zu begründen (»seeing things anew«, 2006: 101). Sein Hauptziel sei erstens, das Konzept der sozialen Beziehung in die Begriffe "kommunikative Anstrengung (effort) zwischen Akteuren" zu übersetzen (2006: 99). Zweitens soll diese kommunikative Anstrengung (endeavor) untersucht werden in Bezug auf die Inhalte, die Organisation von Bedeutungen, die Prozesse und Logiken kommunikativer Ausdrücke sowie die Taktiken von Kommunikation und 
Verstehen, und zwar mit Hilfe des Verfahrens, Erfahrungen von Inkongruenz experimentell zu induzieren (ibid.). Schon als 31-jähriger Graduate Student hat Garfinkel also die Zielsetzung der Ethnomethodologie und das Verfahren der incongruity experiments entwickelt.

Wie Weber und Parsons setzt auch Garfinkel beim sozialen Handeln an und erörtert, was "soziologisch sehen « überhaupt heißen kann. Als Erstes gilt es die Beziehung des soziologischen Beobachters zu seinen Daten zu konzeptualisieren. Die Welt ist ein Faktum - doch wie ist das möglich? Nach Garfinkel läge es nahe, sich mit dem Bedeutungsgehalt so zentraler Begriffe wie `Existenz`, ’Realität` und `Objektivitä‘ auseinanderzusetzen, doch verzichtet er darauf zugunsten einer phänomenologischen Perspektive: „We shall refer instead to the phenomenological researches of Edmund Husserl, and accept his views with regard to the considerations involved for the scientist who seeks a radical and rational empiricism« (Garfinkel 2006: 102). In der Folge orientiert sich Garfinkel vor allem an den Analysen von Schütz, und zwar an dessen Aufsatz "On Multiple Realities" (Schütz 2003 [1945]), in dem dieser unter anderem die Akteursorientierung in der Alltagswelt und die Konzeption von Handlungen durch den wissenschaftlichen Beobachter thematisiert. Garfinkel präsentiert zahlreiche Exzerpte und gibt viele andere Passagen in eigenen Worten wieder. Im Mittelpunkt steht die sinnhafte Struktur der sozialen Welt oder, wie er später sagen wird, das >Problem der Bedeutung` (the problem of meaning) (Garfinkel 2002). Im Unterschied zum Behaviorismus, aber auch zu jenen soziologischen Ansätzen, die soziale Bedeutungen durch ein intersubjektiv geteiltes Symbolsystem gesichert sahen, betont Garfinkel im Anschluss an Schütz die Vielfalt der sinnhaft vorinterpretierten Alltagswelt und die Relevanz interpretativer Akte.

Während sich Schütz in der egologischen Perspektive weitgehend mit den Gegebenheitsweisen der sozialen Welt in der subjektiven Erfahrung beschäftigt, zielt Garfinkel von Anfang an auf eine empirische Erforschung sozialer Kommunikation aus der Beobachterperspektive. Die Phänomenologie ist für ihn nur insoweit von Interesse, als sie ihm für dieses Unterfangen dient. Schütz hat die Beobachterperspektive eingehend analysiert, sowohl was das Fremdverstehen durch ein alltagsweltliches alter ego als auch was die sozialwissenschaftliche Modellbildung durch einen wissenschaftlichen Beobachter anbelangt. Auch »den Mechanismus der Kommunikation vom Standpunkt des Interpretierenden aus« hat er ausführlich beschrieben (2003: 194). Das alter ego kommuniziert einen Gedanken, indem es Wort an Wort, Satz an Satz, Absatz an Absatz reiht, während ego diese Wirkhandlungen fortlaufend interpretiert. Zur Deutung aufgegeben ist dabei das gesamte Ausdrucksfeld, also nicht nur die Sprechhandlungen, sondern auch die Konnotationen und der Kommunikationskontext, der Tonfall der Stimme des Sprechers, sein Gesichtausdruck und seine Gesten, ferner auch bloßes Verhalten und sogar bloße Reflexe, also wesentlich aktuelle Erlebnisse ohne subjektiven Sinn (Schütz 2003: 195f).

Garfinkel $(1967,2006)$ nutzt diese Analysen, um ein soziologisches Programm aus Beobachterperspektive zu entwerfen. In der Kommunikation sind die Akteure mit einem gemeinsamen Sense-making beschäftigt, das sowohl aus Anzeige- als auch aus Deutungsakten besteht. Aufgabe der Ethnomethodologie ist es nach Garfinkel, dieses konzertierte Sense-making in seinem sequenziellen Ablauf zu untersuchen. Denn durch die Art, wie Akteur B auf eine Kommunikation von Akteur A reagiert, bringt er zum Ausdruck, wie er diese Kommunikation verstanden hat, und in der nächsten Sequenz macht Akteur A deutlich, wie er die Reaktion von B verstand (und ob er sich beispielsweise richtig oder falsch verstanden fühlt). Was nicht kommunikativ zum Ausdruck gebracht wird, kann auch nicht verstanden werden. Für Garfinkels Forschungsinteresse ist es daher völlig irrelevant, was im subjektiven Bewusstsein bzw. im Kopf der Interagierenden vor sich geht - nur, was in der Kommunikationssituation beobachtbar ausgedrückt wird, ist Gegenstand der Analyse. Später bringt Garfinkel (1963: 190) dies folgendermaßen auf den Punkt:

»I shall exercise a theorist's preference and say that meaningful events are entirely and exclusively events in a person's behavioral environment ... Hence there is no reason to look un- 
der the skull since nothing of interest is to be found there but brains. The skin of the person will be left intact. Instead questions will be confined to the operations that can be performed upon events that are sscenic to the person."

Srubar $(1988,2007)$ hat sorgfältig herausgearbeitet, dass Schütz’ Lebenswelt zwei Pole aufweist, nämlich einen subjektiv zentrierten und einen intersubjektiven; durch die Sinnklammer der appräsentativen Systeme bleiben sie vermittelt. Sozialität fundiere Subjektivität, und zwar nicht nur in dem Sinne, dass die kulturellen Ausdrucks- und Deutungsschemata einer Gruppe gesellschaftlich vorgegeben und vom Individuum als fraglose, selbstverständliche Wissensbestände angeeignet werden, sondern auch in dem Sinne, dass die Konstitution der objektiven Lebenswelt nicht im subjektiven Bewusstsein, sondern - nunmehr in einem ontologischen Sinne - in der Interaktion der Wirkensbeziehung, der "Urzelle mundaner Sozialität", lokalisiert werden müsse. Damit hat Srubar die Matrix definiert, in der auch die Ethnomethodologie verortet werden kann: Garfinkel setzt am pragmatischen Pol der Lebenswelt an, und dieser kann beobachtet werden.

\section{Elimination anthropologischer Prämissen}

Da es Husserl nicht gelungen war, die Intersubjektivität transzendentalphänomenologisch zu begründen, verzichtete Schütz schon früh auf die epoché, d. h. auf die Einklammerung der Geltungssetzungen der natürlichen Einstellung, und betrieb fortan eine Mundanphänomenologie. Sie begann mit der "Generalthesis des alter ego«, also mit der Annahme der existenziellen Gegebenheit des Anderen im Sinnhaften Aufbau der Welt (Schütz 2004) und setzte sich in zahlreichen anthropologischen Annahmen fort. War das "transzendentale ego" bei Husserl nicht einfach mit dem Ich eines Menschen gleichzusetzen - zumindest auf seinem Totenbett hielt er die Unsterblichkeit des transzentalen egos durchaus für möglich (Schütz 1977: 44) -, bezieht sich das mundane ego bei Schütz nicht nur auf ein erkennendes Subjekt als solches, sondern auch auf konkrete Menschen in ihrer Leiblichkeit, Sozialität und Historizität. ${ }^{2}$ Dies manifestiert sich in zahlreichen Konzepten, wie etwa "menschliche Handlungen«, »biographiespezifischer Wissensvorrat", "Lebenspläne", die "Fundamentalangst" des Menschen bzw. seine "Angst vor dem Tod", aus der die "Systeme von Hoffnungen und Befürchtungen, von Wünschen und Befriedigungen, von Chancen und Risiken" entspringen (Schütz 2003: 204); oder etwa in der Aussage, dass Intersubjektivität und Wir-Beziehung alle anderen Kategorien des Menschseins fundiere, "solange Menschen von Müttern geboren werden" (Schütz 1971: 116).

Hier weicht Garfinkel nun deutlich von Schütz ab: Er streicht sämtliche anthropologischen Prämissen. Akteure sind in der ethnomethodologischen Perspektive keine konkreten Individuen oder Menschen aus Fleisch und Blut, die aufgrund ihrer Intentionen bestimmte Handlungen vornehmen, in verschiedenen Situationen unterschiedliche Rollen spielen und ein Selbst oder eine persönliche Identität, eine Biographie und Pläne für die Zukunft haben. Akteure sind vielmehr Sinnkonstruktionen, die im jeweiligen Kommunikationszusammenhang erst erzeugt werden. Zugespitzt formuliert: Für die Ethnomethodologie existieren keine menschlichen Akteure, sondern nur Handlungen. Handlungen werden nicht von Akteuren erzeugt, sondern Akteure durch Handlungen. Dieser Punkt kann kaum übertont werden, da mit dem Akteursbegriff oft unversehens der methodologische Individualismus konnotiert oder zumindest die

1 Mehr noch als Schütz, distanziert sich Garfinkel aber auch deutlich von verschiedenen Prämissen des Pragmatismus. Vgl. seine Auseinandersetzung mit James, Mead, Peirce und Dewey, zusammengefasst bei Rawls (2006: 54-81).

2 Vgl. dazu Knoblauch und Reichertz in diesem Band. 
ontologische Annahme der natürlichen Einstellung unterstellt wird, dass Akteure Menschen sind, die Situationen betreten und in diesen handeln und kommunizieren.

Akteure sind keine Personen (Garfinkel 2006: 186), sondern durch situierte Handlungen konstituierte Identitäten. Später führt Garfinkel (1967: 76) den Begriff des »Mitglieds« ein, den er im Anschluss an Parsons im Sinne einer Mitgliedschaft in einem Kollektiv versteht. Damit ist die Gefahr, Akteure als Individuen oder Menschen zu reifizieren, etwas gemildert. $\mathrm{Ob}$ jemand Mitglied ist, wird jedoch nicht wie bei Parsons vom (wissenschaftlichen) Beobachter entschieden, sondern von den anderen Mitgliedern des betreffenden Kollektivs: Mitglied ist man so lange, als einem die anderen als "Mitglied « akzeptieren. Und das lässt sich anhand ihrer Handlungen beobachten.

Mit den Personen verabschiedet Garfinkel (2006, 1967) auch andere reifizierende Konstrukte, wie beispielsweise die Vorstellung, Akteure seien gleichsam Behälter von Wissen und Motiven. Er stellt Konzepte wie »subjektive Wissensvorräte« oder "geteiltes Wissen« (shared knowledge) pointiert in Frage und konzentriert sich ausschließlich auf das Wissen im Sinne von "Wie?» Wie wird Kommunikation bewerkstelligt? Wie entsteht der Eindruck, man habe sich erfolgreich verständigt? Woran erkennt man Missverständnisse oder differente Perspektiven? Wie gibt sich eine Frau erfolgreich als »Frau« zu erkennen und Geschworene als "Geschworene«? 1953 führt Garfinkel den Begriff der "Ethnomethoden" und der "Ethnomethodologie» ein, und seither spricht er von Mitgliedermethoden (members methods). Die zugrunde liegende Idee ist so einfach wie plausibel: Das Alltagsleben ist sinnhaft geordnet. Diese Ordnung wird von den Mitgliedern laufend hergestellt, dargestellt und einander angezeigt und dadurch verstehbar gemacht. Folglich müssen die Akteure irgendwelche Methoden beherrschen, um dies zu bewerkstelligen (Garfinkel 1974).

Später wird Garfinkel (2002) von »embodied practices« sprechen. Mit dem Begriff »Praktiken« werden zum einen die handlungstheoretischen Konnotationen vermieden, die mit »Handeln« und »Handlung « unweigerlich mitschwingen. Zum anderen wird die Leiblichkeit eingeführt - was Garfinkels Auseinandersetzung mit Merleau-Ponty widerspiegelt -, allerdings nicht im Sinne der Leiblichkeit von "Akteuren«, sondern im Sinne von verkörperten Praktiken. Beobachtet man Praktiken, so beobachtet man immer körperliche Bewegungen von Akteuren. Im Fokus der Ethnomethodologie stehen Praktiken in ihrem Vollzug, und sie konstituieren auch die situierte Identität der beteiligten Mitglieder. Von »Akteuren« spricht Garfinkel heute noch, aber immer in der beschriebenen eingeschränkten Bedeutung. Um ihnen den Nimbus des agens zu nehmen, ist auch immer mehr von »self-organizing settings« die Rede.

\section{Kognitive Stile und das Problem sozialer Ordnung}

Schon in Garfinkels Frühschriften sind Akteure nicht konkrete Entitäten, sondern »Symbolbehandler " (symbol treaters). Es gilt nach Garfinkel (2006: 109ff) daher die Bedingungen zu spezifizieren, unter denen Symbole behandelt werden. Schütz (2003) nannte dies den »kognitiven Stil«. Dieser steht nach Garfinkel dann fest, wenn die empirischen Spezifikationen der folgenden Konzepte bestimmt sind: (1) der spezifische Modus der Aufmerksamkeit aufs Leben; (2) die Epoché; (3) die spezifische Form der Sozialität; (4) die spezifische Form der Spontaneität; (5) der spezifische Modus des Zeitbewusstseins; und (6) die spezifische Form der Erfahrung des Selbst. Schütz (2003) hat anhand dieser grundlegenden Charakteristika die Alltagswelt, die verschiedenen Welten der Phantasievorstellungen, die Welt der Träume und schließlich die Welt der Wissenschaft beschrieben. Garfinkel will nun dieselben Merkmale verwenden, um soziale Settings zu beschreiben. Beispielhaft fragt er, worin der kognitive Stil eines Wächters der Universitätsbibliothek besteht (2006: 110ff). Um diesen zu explizieren, muss man weder ins subjektive Bewusstsein des Bibliothekswächters eindringen noch eine egologische Analyse 
durchführen. Vielmehr gilt es nach Garfinkel zu fragen, wie die einzelnen Charakteristika des kognitiven Stils aufgrund beobachteter Praktiken empirisch spezifiziert werden können. Dazu müssen neue Methoden entwickelt werden.

In Anlehnung an Parsons (bzw. an Hobbes) akzeptiert Garfinkel das Problem der sozialen Ordnung als die Grundfrage der Soziologie. Bereits in "Seeing Sociologically» konstatiert er, dass Parsons zwar radikal in der Problemstellung, jedoch nicht radikal genug in seinen theoretischen Analysen gewesen sei (Garfinkel 2006: 137). Die invarianten Strukturen, die Husserls Phänomenologie expliziert, seien von wesentlich anderer Art als der theoretische Bezugsrahmen, den Parsons in Structure of Social Action (1968 [1937]) vorlegte. Dieser eigne sich zwar, wie aus Schütz' Weber-Analyse hervorging, zur Applikation auf teleologische Handlungen, nicht aber für expressive, z. B. intime Aktivitäten. In seiner über 600seitigen Dissertation, The Perception of the Other, stellt Garfinkel (1952) nach einigen metatheoretischen Erörterungen die konstitutiven Prämissen des Parsons'schen Theorieansatzes und jene der Schützschen Lebensweltanalyse einander als zwei alternative Paradigmen zur Erklärung sozialer Ordnung gegenüber, als "Korrespondenztheorie vs. Kongruenztheorie», und unterscheidet sie anhand von sechs Kriterien (1952: 90-150) ${ }^{3}$. Obwohl Parsons sein Doktorvater war, entschied er sich nach diesem Vergleich für die Kongruenztheorie von Schütz und führte sein erstes Inkongruitätsexperiment durch (Garfinkel 1952: 391-602). Die Idee war folgende: Betrachtet man eine soziale Situation als eine sinnhafte Ordnung von Objekten, so stellt sie einen geschlossenen Sinnbereich dar, der auf dem Wirken eines kognitiven Stils beruht, den die beteiligten Mitglieder miteinander teilen. Setzt man nun eine dieser konstitutiven Annahmen des kognitiven Stils außer Kraft, müsste - so die Hypothese - die Ordnung zusammenbrechen.

Aus der Korrespondenz zwischen Schütz und Garfinkel geht hervor, dass Schütz daran zweifelte, ob zwischen den theoretischen Entscheidungen von Parsons und ihm wirklich derart fundamentale Unterschiede existieren. Auch war er sich nicht sicher, ob er den grundsätzlichen Unterschied zwischen dem, was Garfinkel »Korrespondenztheorie« respektive »Kongruenztheorie» nannte, begriffen hat und ob es sich wirklich um Unterschiede auf der Ebene empirischer Untersuchungen handelt (vgl. Psathas im Druck). In der Tat hat Schütz in seiner Korrespondenz mit Parsons (Schütz \& Parsons 1977) den Standpunkt vertreten, dass die phänomenologische Analyse der »subjektiven Perspektive« für die soziologische Theorie von Parsons gleichsam einen Bezugsrahmen darstelle, dass Parsons aber seinen Theorieansatz nicht ändern müsse. Die phänomenologisch beschriebenen Strukturen der Lebenswelt sollten vielmehr deutlich machen, welche Sinntransformationen soziologische Theorien vornehmen, wenn sie die subjektive Handlungsorientierung in Homunculus-Modelle übersetzen.

\section{Adäquanz soziologischer Beschreibung: Zurück zu den Phänomenen!}

Schütz hat eine Reihe methodologischer Postulate für sozialwissenschaftliche Theoriekonstruktionen aufgestellt, von denen das Postulat der subjektiven Interpretation und das Postulat der Adäquanz die wichtigsten sind. Das Postulat der subjektiven Interpretation verlangt, dass Generalisierungen und Idealisierungen auf hohem Abstraktionsniveau lediglich als eine Art intellektueller Kurzschrift betrachtet werden und jederzeit auf das Forschungsniveau der individuellen menschlichen Tätigkeit und den subjektiven Sinn, den ein Handeln oder sein Ergebnis für den Handelnden gehabt hat, transformiert werden können (2004a). Das Postulat der Adäquanz verlangt, dass die Konsistenz der Konstruktionen zweiter Ordnung (d. h. der Sozialwissenschaften) mit den Konstruktionen erster Ordnung (des Alltagsdenkens) konsistent sind, dass also "jeder Begriff in einem wissenschaftlichen Modell menschlichen Handelns [...] so kon-

3 Für eine Kurzübersicht vgl. Psathas (im Druck). 
struiert sein (muss), dass eine innerhalb der Lebenswelt durch ein Individuum ausgeführte Handlung, die mit der typischen Konstruktion übereinstimmt, für den Handelnden selbst ebenso verständlich wäre wie für seine Mitmenschen, und das im Rahmen des Alltagsdenkens« (Schütz 2004a: 194). Tiefer in die Details der typisierenden Methode einzudringen, hielt Schütz daher für "die wichtigste Aufgabe einer jeden Handlungstheorie« (1972: 21). Zum einen gilt es die Sinnverschiebungen sorgfältig zu beachten, die bei einer Modifikation des Relevanzsystems und der Transformation von abstrakten zu konkreten Begriffen und von anonymen zu personalen Idealtypen auftreten. Zum anderen macht Schütz deutlich, dass zwischen den sozialwissenschaftlichen Homunculi und der lebensweltlichen Erfahrung von Akteuren eine unaufhebbare Differenz besteht, da die idealtypischen Konstruktionen die typentranszendenten Aspekte nicht erfassen.

Garfinkel fasst das Postulat der Adäquanz wesentlich radikaler als Schütz. Er nimmt dessen Forderung ernst, die subjektive Akteursperspektive bis in ihre subtilsten Sinnabschattungen zu rekonstruieren. Die adäquate Deskription einer sozialen Situation verlangt, die beobachtbaren Praktiken in all ihren Details zu beschreiben und dadurch zu explizieren, wie eine sinnhafte soziale Ordnung erzeugt, kommunikativ angezeigt und dadurch verstehbar (intelligible) gemacht wird. Garfinkel löst damit das ursprüngliche phänomenologische Diktum »Zurück zu den Phänomenen!« ein, indem er der Ethnomethodologie zur Aufgabe macht, Kommunikationsvollzüge in sozialen Situationen in all ihren Einzelheiten genau zu beobachten und zu beschreiben. Noch heute gehört es zum üblichen Diskurs von Ethnomethodologen, beim Anblick aufgezeichneter Daten zu fragen: „Was ist das Phänomen?«. Allerdings geht es nicht darum, die Erscheinungsweisen von Phänomenen im subjektiven Bewusstsein des Beobachters zu analysieren, sondern zu erläutern, aufgrund welcher kommunikativer Praktiken soziale Situationen geordnet und verständlich gemacht werden. Weder der kognitive Stil noch Reflexions- und Verstehensakte müssen im Bewusstsein gesucht, sondern können unmittelbar in Kommunikationssituationen beobachtet werden: Wie beispielsweise Mitglieder indexikale Ausdrücke in ihrer Reflexivität sequenziell erschließen und mittels der dokumentarischen Methode der Interpretation ein Sinnmuster erkennen, lässt sich am konkreten Datenmaterial zeigen (Garfinkel 1967).

Allerdings wirft dies sofort die Frage auf, was denn eigentlich am empirischen Material gezeigt werden kann. Die Reflexion darauf, wie sich aus der Beobachterperspektive schlüssig aufweisen lässt, woran sich die Akteure faktisch orientieren, blieb im Vergleich zu den Schütz'schen Analysen des Fremdverstehens relativ dürftig, und einige Ethnomethodologen und Konversationsanalytiker gebärdeten sich manchmal etwas empirizistisch. Solange man Alltagssituationen untersuchte, die "jedermann" versteht, wurde auch bei den Beobachtern die Kompetenz, beobachtete Situationen »richtig« interpretieren zu können, einfach vorausgesetzt. Als Garfinkel und seine Kollegen aber mit den Studies of Work begannen (Garfinkel 1986), sprach er zunehmend vom "unique adequacy criterion «: Der Beobachter muss ein kompetentes Mitglied der untersuchten Situation sein, um diese adäquat verstehen zu können. Waren die frühen Ethnomethodologen fast ausschließlich Soziologen, rekrutierte Garfinkel später Mathematiker, Physiker, Juristen und andere, da nur sie über die Kompetenz verfügten, die Arbeit solcher Professionsangehöriger adäquat zu verstehen. Neuerdings geht Garfinkel (2002) aber noch einen Schritt weiter, indem er "Adäquanz« nicht mehr an eine Beschreibung koppelt, sondern sie in der erfolgreichen »Instruktion« erblickt.

\section{Die Entwicklung der Ethnomethodologie}

Heute liegt ein umfangreicher Corpus ethnomethodologischer Arbeiten vor. Im Laufe der Weiterentwicklung sind gegenüber den frühen Schriften Garfinkels zahlreiche konzeptuelle Änderungen und Fokusverschiebungen beobachtbar, die hier nicht nachgezeichnet werden 
können. ${ }^{4}$ In Bezug auf die Phänomenologie sollen jedoch vier Punkte erwähnt werden: Erstens hat sich die Ethnomethodologie nicht nur als Alternative zu Parsons' Strukturfunktionalismus, sondern zur gesamten konventionellen Soziologie profiliert. Indem diese mit Umschreibungen (glosses), also mit monothetischen Konstrukten operiert und damit die konstitutiven kommunikativen Akte der Erzeugung sozialer Ordnung überblendet, verwechselt sie Gegenstand und Mittel, setzt also in ihren Erklärungen gerade das voraus, was sie eigentlich erklären sollte (Zimmerman \& Pollner 1976). ${ }^{5}$ Denn die Anwendung der traditionellen theoretischen Konzepte, wie Norm, Rolle, Zeichen usw., ist nur mit Hilfe von Interpretationsprozeduren möglich, die jeweils als unreflektierte und unthematisierte Ressourcen in die soziologische Beschreibung konkreter Siuationen mit einfließen. Die konventionelle Soziologie wendet somit dieselben Methoden an wie die Akteure im Alltagsleben, wenn sie soziale Situationen beschreiben. Aufgrund dieser Gemeinsamkeiten wird sie von Garfinkel (1967) als »folk sociology" bezeichnet. Die Ethnomethodologie will demgegenüber einen ganz anderen Weg gehen, indem sie das Problem der Ordnung auf einer grundsätzlicheren Ebene ansetzt und die Methoden aufzudecken sucht, mittels derer gesellschaftliche Fakten konstituiert und raccountable‘, also erkennbar, verstehbar, beschreibbar, berichtbar und erklärbar gemacht werden (Garfinkel 1967: vii).

Zweitens: Ein weiteres wichtiges Thema, das Schütz in der Auseinandersetzung mit Parsons angesprochen und später (2004a) weiter ausgearbeitet hat, war der Unterschied zwischen der Rationalität auf der Ebene wissenschaftlicher Konstruktionen und jener im Alltagsleben. Schütz' Argumente wurden von Parsons nicht verstanden (Schütz \& Parsons 1977) und haben Garfinkel (1967: 262ff) zu einer ausführlichen Erörterung von Alltagsrationalitäten und wissenschaftlichen Rationalitäten veranlasst. ${ }^{6}$ Die rationalen Merkmale praktischer Handlungen als kontingente, andauernde Hervorbringungen von organisierten, kunstvollen Praktiken des Alltagslebens zu untersuchen, ist fortan ein erklärtes Ziel der Ethnomethodologie (Garfinkel 1967: 11).

Drittens: Eine weitere intensive Auseinandersetzung mit Schütz fand im Rahmen der "Studies of Work" statt, als sich Michael Lynch (1993) im Rahmen seines Postulats, Epistemologie zum Gegenstand empirischer ethnomethodologischer Analysen zu machen und damit eine neue Soziologie wissenschaftlichen Wissens zu begründen, für eine Revision Schützscher Annahmen stark machte. In einer kürzlich veröffentlichten Debatte wirft Dennis (2004) ihm vor, er postuliere einen epistemologischen und methodologischen Bruch innerhalb der Ethnomethodologie: "Garfinkel's later works are argued to be superior to their earlier, 'protoethnomethodological, counterparts to the extent that they no longer rest on Schutz's flawed and scientistic approach to methodological rigour" (2004: 1). Dennis' Gegenthese lautet, die Kritik an Schütz sei unhaltbar und Garfinkels Arbeiten wiesen durchaus Kontinuität auf. Lynch (2004) antwortet in einer Replik, "Misreading Schutz«, und Sharrock (2004) ergänzt, "What Garfinkel makes of Schutz«. Diese Debatte erhellt vor allem die innerethnomethodologischen Auseinandersetzungen der jüngeren Zeit, fördert aber keine neuartigen Aspekte in Bezug auf das Verhältnis von Schütz und Garfinkel zutage. Was Schütz betrifft, sprechen trotz der geäußerten Kritik alle drei Autoren nach wie vor mit großem Respekt von ihm.

Viertens: Die eigentliche Herausforderung der Ethnomethodologie lag von allem Anfang an darin, Methoden zu entwickeln, mittels derer die Ethnomethoden erfolgreich erforscht werden konnten. Garfinkel entwickelte viele Inkongruitätsexperimente, manchmal auch »breaching experiments" genannt, und beruft sich dabei ausdrücklich auf Schütz (Garfinkel 1967: 37). Diese Experimente sind keine Experimente im klassischen Sinn, sondern "Demonstrationen", welche die Hintergrundserwartungen deutlich machen sollen, mit denen Akteure in der natürlichen Einstellung in konkreten sozialen Settings operieren. Daneben sammelte Garfinkel auch Daten

4 Für einen aktuellen Überblick vgl. Eberle (2007a, 2007b).

5 Diese Kritik trifft auch die Symbolischen Interaktionisten sowie Goffman (vgl. Rawls 2006).

6 Für eine vertiefte Auseinandersetzung vgl. Eberle (1984: 469ff). 
per Interview - z. B. in seiner Studie über die Transsexuelle Agnes (1967: 116-185) -, insbesondere aber mittels ethnographischer Beobachtungen. Seit seiner Zusammenarbeit mit Harvey Sacks und dem Aufschwung der Konversationsanalyse kamen zunehmend auditive und schließlich audiovisuelle Aufzeichnungen zum Einsatz, die den Vorteil hatten, dass dieselbe sequenziell organisierte Szene immer wieder beobachtet und immer eingehender analysiert werden konnte.

Der Beitrag der Ethnomethodologie an die Methoden der qualitativen Sozialforschung war enorm. Die Akribie der Datenaufzeichnungen und Transkriptionen, die Detailbesessenheit der Analysen und die Selbstdisziplin, datengeleitet und nicht theoriegeleitet vorzugehen, also nichts an die Daten heranzutragen, was diese nicht selbst belegen, ist unübertroffen geblieben. Wenn man in Rechnung stellt, wie lange die an Schütz orientierten empirischen Verfahren - wie etwa das narrative Interview oder die sozialwissenschaftliche Texthermeneutik - sich vorab auf Interviewmaterial beschränkten, war die Ethnomethodologie bahnbrechend. Ihre Radikalität, nur Beobachtungsdaten aus natürlichen Settings zuzulassen und Interviewdaten als bloße Narrationen (glosses) zu disqualifizieren, legt ihr allerdings auch Grenzen auf: Erstens beschränkt sich der Fokus auf Interaktionssituationen, zweitens auf kurze fragmentarische Sequenzen und drittens auf die Akteursorientierung, insoweit sie durch Beobachtung rekonstruiert werden kann. Wie die Erfahrung zeigt, sind aber auch ethnomethodologische Analysen nicht über allen Zweifeln erhaben, ob die Akteure sich tatsächlich an dem orientieren, was die wissenschaftlichen Beobachter ihnen zuschreiben.

Im Laufe dieser Entwicklung haben sich Garfinkel und seine Schüler immer mehr auf die Empirie konzentriert, und ab den 1970er Jahren konvertierten immer mehr Ethnomethodologen zur Konversationsanalyse, weil diese die überzeugendsten Resultate zu erbringen schien. Immer mehr setzte sich die Überzeugung durch: Über Ethnomethodologie spricht man nicht, Ethnomethodologie betreibt man! Auch Garfinkel zeigte sich immer weniger auskunftswillig und widerstand zunehmend theoretischen Erörterungen oder Theorievergleichen, operierten diese doch alle mit relativ abstrakten Begrifflichkeiten, also mit "glosses", statt dass sie die Antworten in einer Analyse konkreter empirischer Daten suchten. Garfinkel zitiert zwar auch in seinen neuesten Publikationen (2002) noch Phänomenologen, nun eher Gurwitsch ${ }^{7}$ und Merleau-Ponty statt Schütz, empfiehlt aber explizit die Strategie des "misreading" (Garfinkel 2002: 112). Damit meint er nicht ein "unkorrektes«, aber ein alternatives, kreatives Lesen. Die klassischen Texte sollen nicht philologisch ausgelegt und anhand theoretischer Kriterien diskutiert werden, sondern der Inspiration zur empirischen Analyse konkreter Daten, zum »seeing sociologically«, dienen. Die Aversion gegen systematische Theoriedebatten hat allerdings, verstärkt durch die eher kryptische Ausdrucksweise Garfinkels, auch zu einem gewissen Obskurantismus geführt, den die Ethnomethodologie nie ganz losgeworden ist.

\section{Konklusion: Phänomenologie und Ethnomethodologie}

Die Phänomenologie war für die Genese der Ethnomethodologie konstitutiv. Garfinkel hat seine Ideen in enger Anlehnung an Schütz und auch an Gurwitsch und Husserl sowie später an Merleau-Ponty entwickelt. Von Anfang hat er sie nicht als Protosoziologie interpretiert, sondern als alternativen soziologischen Ansatz zur Analyse der sozialen Ordnung. Die Ethnomethodologie rekurriert daher nicht auf das subjektive Bewusstsein der Handelnden, sondern beobachtet Kommunikationsakte, anhand derer sie sowohl die Anzeigehandlungen als auch die Interpretationsakte der beteiligten Mitglieder in ihrem sequenziellen Ablauf rekonstruiert. Es geht wie bei Schütz um die subjektive Akteursorientierung, aber nur insoweit sie sich durch

7 Garfinkel (2002) spricht von den "phänomenalen Feldmerkmalen von sozialen Dingen« und bezieht sich dabei auf Gurwitschs Idee der Gestalt (Rawls 2002: 14). 
empirische Beobachtung erschließen lässt. Im Laufe ihrer Entwicklung hat sich die Ethnomethodologie von ihren phänomenologischen Fundamenten etwas entfernt und sich immer dezidierter empirischen Analysen gewidmet. Die methodischen Verfahren, die sie dabei entwickelt hat, waren wegweisend und gehören heute zum festen Methodenarsenal der qualitativen Sozialforschung. Vergleicht man die ethnomethodologischen Studien mit den Arbeiten jener Kolleginnen und Kollegen, die Husserl- und Schütz-Exegese betreiben oder sich mit theoretischen und methodologischen Erörterungen befassen, so leben die Ethnomethodologen wohl am ehesten dem Diktum nach, mit dem die Phänomenologie seinerzeit ins Feld zog: "Zurück zu den Phänomenen!« Die Ethnomethodologie lehrt den akribisch exakten, detailverliebten Blick auf die empirischen Gegebenheitsweisen sozialer Phänomene. Dabei unterstellt sie keine universalen Strukturen subjektiver Weltorientierung a priori, sondern fragt im Gegenteil danach, wie das Spezifische, Kennzeichnende konkreter sozialer Settings hervorgebracht wird. Adäquate Analyse heißt für Ethnomethodologen, die konstitutive Bedeutung jedes Details einer sozialen Situation zu erkennen und zu beschreiben und, im Fall von Arbeitssettings, Neumitglieder kompetent instruieren zu können.

Interpretiert man umgekehrt die phänomenologische Lebensweltanalyse als Protosoziologie, so kann selbstverständlich auch die Ethnomethodologie, analog zu jeder anderen Art sozialwissenschaftlicher Theoriekonstruktion und Forschung, in der Strukturmatrix verortet und deskriptiv analysiert werden. Die Lebensweltanalyse macht deutlich, was Ethnomethodologen tun. Seit ihren Anfängen zeigt die Ethnomethodologie deutliche Affinitäten zur pragmatischen Lebenswelttheorie: Beide gehen davon aus, dass die Sozialwelt durch Wirkhandlungen konstituiert wird und eine soziologische Analyse bei den Appräsentationsystemen anzusetzen hat. Die Lebenswelttheorie geht dabei eher theoretisch-reflexiv vor, während die Ethnomethodologie empirisch-reflexiv verfährt. Beide haben das Potential, einander zu ergänzen: Die Lebensweltanalyse liefert eine protosoziologische Konstitutionstheorie, die Ethnomethodologie verzichtet darauf und führt die Konstitutionsanalysen anhand konkreter sozialer Settings empirisch durch. Während die Lebenswelttheorie auf die universalen Strukturen der Weltorientierung zielt, beschäftigt sich die Ethnomethodologie mit den »haecceitas«, also mit dem Spezifischen und Einmaligen, der "just-thisness" von Phänomenen als Ausdruck sozialer Ordnung (Garfinkel 2002: 99). Beide sind komplementär, fordern einander aber auch heraus: die Lebenswelttheorie durch das breite Spektrum und die theoretische Schärfentiefe ihrer Reflexionen, die Ethnomethodologie durch ihre filigranen empirischen Nanoanalysen. Beide tun gut daran, voneinander zu lernen.

\section{Literatur}

Berger, Peter L. \& Thomas Luckmann (1969), Die gesellschaftliche Konstruktion der Wirklichkeit, Frankfurt a.M.: Fischer

Dennis, Alex (2004), Lynch on Schutz and Science: Postanalytic Ethnomethodology Reconsidered, Theory \& Science 5.1, auf: http://theoryandscience.icaap.org/content/vol5.1/dennis.html (01.01.08)

Eberle, Thomas (1984), Sinnkonstitution in Alltag und Wissenschaft. Der Beitrag der Phänomenologie an die Methodologie der Sozialwissenschaften, Bern \& Stuttgart: Haupt

Eberle, Thomas (2007a), Ethnomethodologie, in: Renate Buber \& Hartmut Holzmüller (Hg.), Qualitative Marktforschung. Konzepte - Methoden - Analysen, Wiesbaden: Gabler, S. 93-109

Eberle, Thomas (2007b), Ethnomethodologie und Konversationsanalyse, in: R. Schützeichel (Hg.), Handbuch Wissenssoziologie und Wissensforschung, Konstanz: UVK, S. 139-160

Garfinkel, Harold (1952), The Perception of the Other, Harvard University: unveröff Diss.

Garfinkel, Harold (1967), Studies in Ethnomethodology, Englewood Cliffs: Prentice-Hall

Garfinkel, Harold (1974), The Origins of the Term „Ethnomethodology`, in: R. Turner: Ethnomethodology. Selected Readings, London: Penguin, S. 15-18 
Garfinkel, Harold (1986), Ethnomethodological Studies of Work, London \& New York: Routledge \& Kegan Paul

Garfinkel, Harold (2002), Ethnomethodology's Program. Working Out Durkheim's Aphorism, Lanham: Rowman \& Littlefield

Garfinkel, Harold (2006 [1948]), Seeing Sociologically. The Routine Grounds of Social Action, Lanham: Rowman \& Littlefield

Heritage, John (1984), Garfinkel and Ethnomethodology, Cambridge: Polity Press

Lemert, Charles (2006), Foreword: The Indexical Properties of Sociological Time, in: Harold Garfinkel: Seeing Sociologically. The Routine Grounds of Social Action, Lanham: Rowman \& Littlefield, S. vii-xiii

Luckmann, Thomas (1979), Phänomenologie und Soziologie, in: W. Sprondel \& R. Grathoff (Hg.), Alfred Schütz und die Idee des Alltags in den Sozialwissenschaften, Stuttgart: Enke, S. 196-206

Luckmann, Thomas (1980), Lebenswelt und Gesellschaft. Grundstrukturen und geschichtliche Wandlungen, Paderborn: Schöningh

Lynch, Michael (1993), Scientific practice and ordinary action, Cambridge: University Press

Lynch, Michael (2004), Misreading Schutz: A Response to Dennis on 'Lynch on Schutz on Sciences, Theory \& Science 5.1, auf: http://theoryandscience.icaap.org/content/vol5.1/lynch.html (01.01.08)

Parsons, Talcott (1968 [1937]), The Structure of Social Action. Vol. 1+2, New York: The Free Press

Psathas, George (im Druck), The correspondence of Alfred Schutz and Harold Garfinkel: What was the 'Terra Incognita and the 'Treasure Island ??, in: L. Embree, G. Psathas, H. Nasu \& I. Srubar (Hg.), Alfred Schutz and His Intellectual Partners, Konstanz: UVK

Psathas, George (2004), Alfred Schutz's Influence on American sociologists and sociology, Human Studies 27, S. $1-35$

Rawls, Anne Warfield (2002), Editors' Introduction, in: Harold Garfinkel: Ethnomethodology's Program. Working Out Durkheim's Aphorism, Lanham: Rowman \& Littlefield, S. 1-64

Rawls, Anne Warfield (2006), Respecifying the Study of Social Order - Garfinkel's Transition from Theoretical Conceptualization to Practices in Details, in: Harold Garfinkel: Seeing Sociologically. The Routine Grounds of Social Action, Lanham: Rowman \& Littlefield, S. 1-97

Schütz, Alfred (1971), Das Problem der transzendentalen Intersubjektivität bei Husserl, in ders., GA III, Studien zur phänomenologischen Philosophie, Den Haag: Martinus Nijhoff, S. 86-118

Schütz, Alfred (1972), Die soziale Welt und die Theorie der sozialen Handlung, in ders., GA II, Studien zur soziologischen Theorie, Den Haag: Martinus Nijhoff, S. 3-21

Schütz, Alfred (1977), Husserl and His Influence on Me, in: Lester Embree (ed.), The Annals of Phenomenological Sociology 2, S. 41-44

Schütz, Alfred (2003 [1945]), Über die mannigfaltigen Wirklichkeiten, in ders., Theorie der Lebenswelt 1, ASW V.1, Konstanz: UVK, S. 177-147

Schütz, Alfred (2004a), Common-sense und wissenschaftliche Interpretation menschlichen Handelns, in: J. Strübing \& B. Schnettler (Hg.), Methodologie interpretativer Sozialforschung. Klassische Grundlagentexte, Konstanz: UVK/UTB, S. 155-97

Schütz, Alfred (2004b), Der sinnhafte Aufbau der sozialen Welt. ASW II., Konstanz: UVK

Schütz, Alfred \& Thomas Luckmann (2003), Die Strukturen der Lebenswelt, Konstanz: UVK/UTB

Schütz, Alfred \& Talcott Parsons (1977), Zur Theorie sozialen Handelns. Ein Briefwechsel, Frankfurt a.M.: Suhrkamp

Sharrock, Wesley \& Bob Anderson (1989), Epistemology: professional scepticism, in: Graham Button, Ethnomethodology and the Human Sciences, Cambridge: University Press, S. 51-56

Sharrock, Wes (2004), What Garfinkel makes of Schutz: The past, present and future of an alternate, asymmetric and inconmmensurable approach to sociology, Theory \& Science 5.1, auf: http://theoryand science.icaap.org/content/vol5.1/sharrock.html (01.01.08)

Srubar, Ilja (1988), Kosmion. Die Genese der pragmatischen Lebenswelttheorie von Alfred Schütz und ihr anthropologischer Hintergrund, Frankfurt a.M.: Suhrkamp

Srubar, Ilja (2007), Phänomenologische und soziologische Theorie, Wiesbaden: VS-Verlag

Zimmerman Don H. \& Melvin Pollner (1976), Die Alltagswelt als Phänomen, in: E. Weingarten, F. Sack \& J. Schenkein (Hg.), Ethnomethodologie, Frankfurt a.M.: Suhrkamp, S. 64-104 
Jürgen Raab - Michaela Pfadenhauer Peter Stegmaier · Jochen Dreher Bernt Schnettler

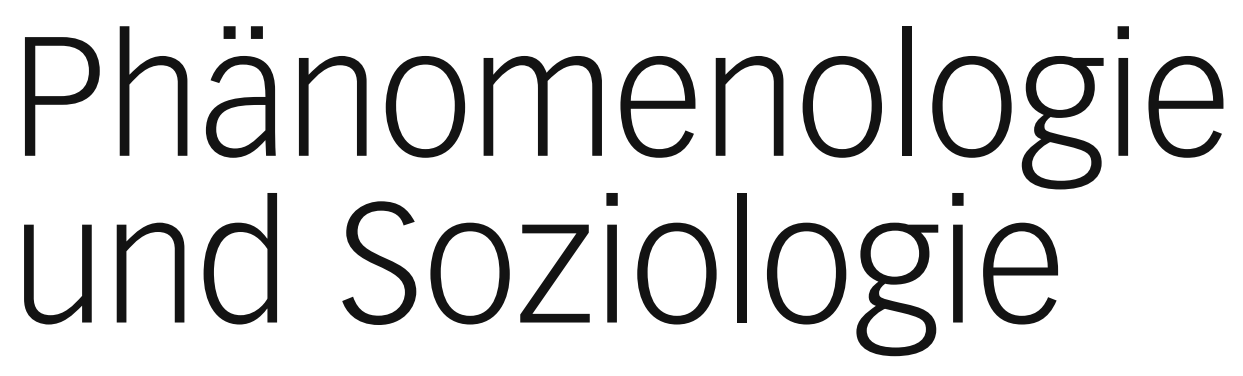

Theoretische Positionen, aktuelle Problemfelder und empirische Umsetzungen 
Bibliografische Information der Deutschen Nationalbibliothek

Die Deutsche Nationalbibliothek verzeichnet diese Publikation in der

Deutschen Nationalbibliografie; detaillierte bibliografische Daten sind im Internet über

$<$ http://dnb.d-nb.de > abrufbar.

\section{Auflage 2008}

Alle Rechte vorbehalten

(c) VS Verlag für Sozialwissenschaften | GWV Fachverlage GmbH, Wiesbaden 2008

Lektorat: Frank Engelhardt

VS Verlag für Sozialwissenschaften ist Teil der Fachverlagsgruppe

Springer Science+Business Media.

www.vs-verlag.de

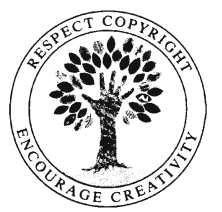

Das Werk einschließlich aller seiner Teile ist urheberrechtlich geschützt. Jede Verwertung außerhalb der engen Grenzen des Urheberrechtsgesetzes ist ohne Zustimmung des verlags unzulässig und strafbar. Das gilt insbesondere für Vervielfältigungen, Übersetzungen, Mikroverfilmungen und die Einspeicherung und Verarbeitung in elektronischen Systemen.

Die Wiedergabe von Gebrauchsnamen, Handelsnamen, Warenbezeichnungen usw. in diesem Werk berechtigt auch ohne besondere Kennzeichnung nicht zu der Annahme, dass solche Namen im Sinne der Warenzeichen- und Markenschutz-Gesetzgebung als frei zu betrachten wären und daher von jedermann benutzt werden dürften.

Umschlaggestaltung: KünkelLopka Medienentwicklung, Heidelberg

Druck und buchbinderische Verarbeitung: Krips b.v., Meppel

Gedruckt auf säurefreiem und chlorfrei gebleichtem Papier

Printed in the Netherlands

ISBN 978-3-531-15428-2 


\section{Inhalt}

\section{Einleitung der Herausgeber}

Phänomenologie und Soziologie. Grenzbestimmung eines Verhältnisses

\section{Theoretische Positionen und Perspektiven}

\section{Thomas Luckmann}

Konstitution, Konstruktion: Phänomenologie, Sozialwissenschaft

Ilja Srubar

Die pragmatische Lebenswelttheorie

Hans-Georg Soeffner

Symbolische Präsenz: unmittelbare Vermittlung - zur Wirkung von Symbolen

\section{Hubert Knoblauch}

Transzendentale Subjektivität. Überlegungen zu einer wissenssoziologischen

Theorie des Subjekts

Jo Reichertz

Das Ich als Handlung oder das handelnde Ich? Nachdenken über einen

lieb gewonnenen Begriff der Phänomenologie

Martin Endreß

Reflexive Wissenssoziologie als Sozialtheorie und Gesellschaftsanalyse.

Zur phänomenologisch fundierten Analytik von Vergesellschaftungsprozessen

\section{Andreas Göttlich}

Sociologia Perennis? Überlegungen zur Problematik prototheoretischer Aussagen in der Soziologie.

Daniel $\check{S}$ uber

Phänomenologie/Lebensphilosophie. Zu einem zentralen Kapitel

im Streit um die Phänomenologie.

Joachim Fischer

Tertiarität. Die Sozialtheorie des "Dritten« als Grundlegung

der Kultur- und Sozialwissenschaften

Ronald Hitzler

Von der Lebenswelt zu den Erlebniswelten. Ein phänomenologischer Weg

in soziologische Gegenwartsfragen

Bernt Schnettler

Soziologie als Erfahrungswissenschaft. Überlegungen zum Verhältnis von Mundanphänomenologie und Ethnophänomenologie

Thomas $S$. Eberle

Phänomenologie und Ethnomethodologie..... 
Armin Nassehi

Phänomenologie und Systemtheorie

Rainer Schützeichel

Transzendentale, mundane und operative (systemtheoretische) Phänomenologie

\section{Problemfelder und aktuelle Debatten}

\section{Nico Lüdtke}

Intersubjektivität bei Schütz - oder: Ist die Frage nach dem Anderen

aus der Phänomenologie entlassen?

Jens Bonnemann

Wege der Vermittlung zwischen Faktizität und Freiheit. Zur Methodologie

der Fremderfahrung bei Jean-Paul Sartre .....

Ingo Schulz-Schaeffer

Soziales Handeln, Fremdverstehen und Handlungszuschreibung.

Gregor Bongaerts

Verhalten, Handeln, Handlung und soziale Praxis

Jürgen Raab

Präsenz und mediale Präsentation. Zum Verhältnis von Körper und technischen

Medien aus Perspektive der phänomenologisch orientierten Wissenssoziologie.

Michael Kauppert

Wie erschließt sich der Erfahrungsraum? Zur Transformation des

Lebenswelttheorems.

Joachim Renn

Emergenz - Das soziologische Problem heterogener Ordnungsebenen

und die Zeit der Phänomenologie.

Peter Stegmaier

Normative Praxis: konstitutions- und konstruktionsanalytische Grundlagen

Dirk Tänzler

Repräsentation Die Brücke zwischen Phänomenologie und Soziologie des Politischen

Thilo Raufer

Politik, Symbolismus und Legitimität Zum Verhältnis von Konstitutionsund Konstruktionsanalysen in der empirischen Forschung

\section{Methodische Reflexionen und Analysen}

Jochen Dreher

Protosoziologie der Freundschaft. Zur Parallelaktion von phänomenologischer und sozialwissenschaftlicher Forschung.

Dariuš Zifonun

Widersprüchliches Wissen. Elemente einer soziologischen Theorie des Ambivalenzmanagements. 


\section{Tobias Röhl}

Symbole des Unfalltodes. Eine mundanphänomenologisch informierte Analyse privater Erinnerungsmale

\section{Sebastian Deterding}

Introspektion. Begriffe, Verfahren und Einwände in Psychologie und Kognitionswissenschaft

\section{Michaela Pfadenhauer}

Doing Phenomenology: Aufgrund welcher Merkmale bezeichnen wir ein Handeln als »kompetentes Organisieren«?

Margarethe Kusenbach

Mitgehen als Methode. Der `Go-Along` in der phänomenologischen Forschungspraxis.....

\section{Thorsten Berndt}

Das beobachtende Interview .Zur relevanztheoretischen Rekonstruktion und innovativen Ergänzung qualitativer Interviews

\section{Ronald Kurt}

Vom Sinn des Sehens. Phänomenologie und Hermeneutik als Methoden visueller Erkenntnis

\section{Anne Honer}

Verordnete Augen-Blicke Reflexionen und Anmerkungen zum subjektiven Erleben des medizinisch behandelten Körpers

Silvana K. Figueroa-Dreher

Musikalisches Improvisieren: Die phänomenologische Handlungstheorie auf dem Prüfstand

\section{Siegfried Saerberg}

Das Sirren in der Dschungelnacht - Zeigen durch

Sich-wechselseitig-aufeinander Einstimmen 